\title{
Facile Synthesis of a Diverse Library of Mono-3-substituted $\beta$-Cyclodextrin Analogues
}

\author{
K. Kellett, B. M. Duggan, M.K. Gilson* \\ Skaggs School of Pharmacy and Pharmaceutical Sciences, University of California San Diego, 9500 Gilman Drive, La \\ Jolla, California 92093-0736, USA
}

\section{Introduction}

Cyclodextrins (CD) are cyclic oligosaccharides, consisting of six ( $\alpha-C D)$, seven $(\beta-C D)$ or eight $(\gamma-C D)$ glucose monomers that together form the shape of a hollow truncated cone (Figure 1). Their $\mathrm{C} 6$ primary hydroxyls rim the narrower face of the cone, while their C2 and C3 secondary hydroxyls are located on the wider face (Figure 1). Together, the hydroxyls make the exterior of cyclodextrin hydrophilic and contribute to its aqueous solubility, while the internal cavity remains hydrophobic, allowing for noncovalent association with lipophilic and amphiphilic molecules. The cyclodextrins have found a number of practical applications, including solubilization of small, lipophilic, drug molecules ${ }^{[1,2]}$, and encapsulation of volatile compounds $^{[3,4]}$. In recent years, cyclodextrins also have been adopted as test cases for computational methods of predicting noncovalent binding affinities. In this context, they represent computationally simple models of more complex protein-ligand molecular recognition systems relevant in drug discovery ${ }^{[5-}$ 7], and are now being used to test and enhance the accuracy of the potential functions that are fundamental to molecular simulations ${ }^{[8,9]}$.The large body of experimental cyclodextrin-guest affinity data available in the literature ${ }^{[7,10-12]}$ is of great value in such applications. However, most of these data are for unmodified cyclodextrins, and hence probe only interactions of guest molecules with the simple glucose monomers of the native host. Enhancing the diversity of experimentally characterized chemical interactions would be of enormous interest in the computational chemistry community, because it would open new possibilities for using host-guest binding data to test and improve potential functions; and applications in other fields, such as chemical separations and pharmaceutical formulations, are also likely.
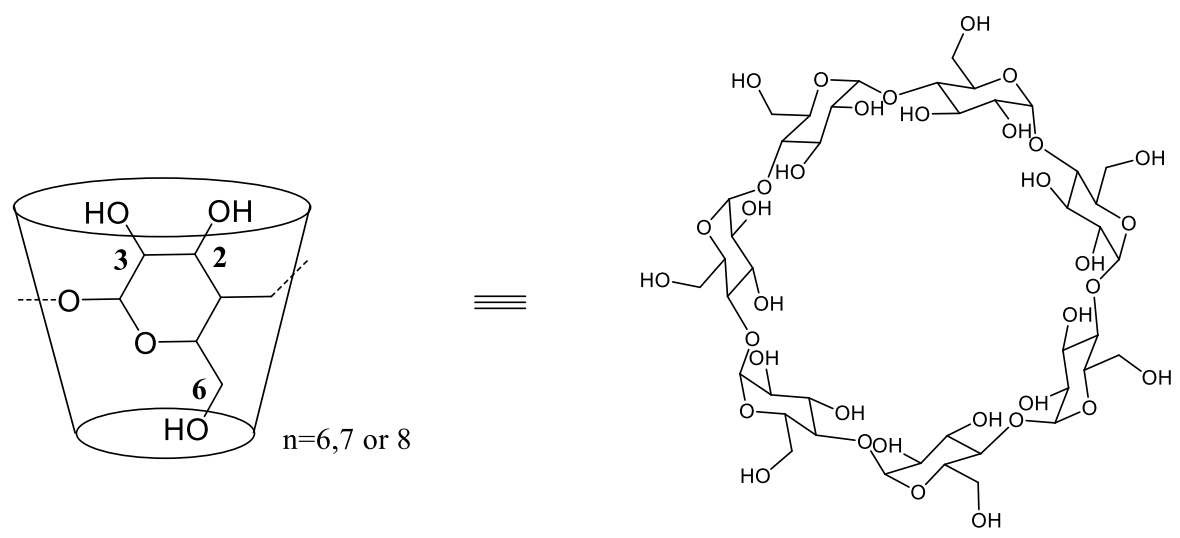

Figure 1. Schematic of truncated cone structure of cyclodextrin (left), and the chemical structure of $\beta$-cyclodextrin (right).

The cyclodextrins can be diversified through the addition of new functional groups at the hydroxyls on either the primary or the secondary face, leading to changes in size, shape and physical properties. For example, although native $\beta-C D$ is not particularly water-soluble $(18 \mathrm{mg} / \mathrm{mL})$, randomly-methylated- $\beta$ cyclodextrin and 2-hydroxypropyl- $\beta$-cyclodextrin have aqueous solubilities of over $500 \mathrm{mg} / \mathrm{mL}^{[13]}$. Of 
particular interest to our group is that derivatization can also modulate the binding affinities of these hosts for small molecule guests that bind in the hydrophobic cavity of $\mathrm{CD}^{[14-17]}$. When modifying the binding affinity and specificity is the goal, it is preferable to derivatize the secondary hydroxyl groups of the host, rather than the primary ones, as derivatizing the secondary hydroxyls typically has a greater effect on binding affinity ${ }^{[18,19]}$. This makes intuitive sense, because bound guest molecules are more likely to protrude through the wider secondary opening, where they can interact with the added substituents, than through the narrower primary opening, as presented in our previous work ${ }^{[20]}$. It may also be advisable to create mono-substituted derivatives, rather than adding multiple substituents, to avoid overcrowding of this entryway and/or a combinatorial explosion of variants in the reaction products ${ }^{[21,22]}$.

However, despite important advances ${ }^{[23-29]}$, the selective addition of substituents at the secondary face, has long posed challenges in synthesis and purification ${ }^{[25]}$. Due to the large number of hydroxyls decorating $C D$, many modification strategies lead to a complex mixture of products, such as randomlymethylated- $\beta-C D$. In addition, modified CDs sometimes aggregate or become insoluble, reducing their effectiveness as host molecules ${ }^{[30-32]}$. Furthermore, selective modification of the secondary face of $\beta-C D$ has involved arduous, low-yielding, reaction procedures, and existing approaches typically require access to purification equipment, such as lyophilizers and centrifuges ${ }^{[1,33-35]}$, not available to all groups interested in synthesizing such derivatives. Efforts at making selectively modified CDs more accessible and cost-effective, such as through the use of mechanosynthetic methods, which may overcome tedious work ups and time consuming purifications, have still afforded only modest reaction yields ${ }^{[35]}$. As a consequence, much of the existing literature focuses on substituting the narrower, primary face of $\beta-C D$, which is easier to functionalize, due to the greater nucleophilicity and reduced steric hindrance of its hydroxyls. However, this expedient yields products that are less interesting from the standpoint of generating diversity in binding affinity and specificity.

Here, we describe synthetic principles for the facile synthesis of diverse cyclodextrins mono-substituted on the secondary face, along with multiple high-yielding, one-pot syntheses, based on these principles, that avoid the need for complicated procedures and equipment. These approaches promise to make modified CDs dramatically more accessible and to allow the discovery of new applications for them.

\section{Results and Discussion}

Reactions designed for primary face modification are typically unsuitable for the less reactive secondary hydroxyls, so our synthetic strategies are specifically optimized for the secondary face. We take mono-3amino- $\beta$-cyclodextrin $\left(3-\mathrm{NH}_{2}-\beta-\mathrm{CD}\right)$ as a starting point. Its synthesis is well documented ${ }^{[26,33]}$, its amino group is more reactive than the hydroxyls, and chemo-selective reactions directed towards the amine eliminate the large number of products that are often produced upon direct functionalization of the hydroxyls. Nonetheless, adoption of this starting material does not resolve critical challenges of reactivity and purification. The synthetic strategies described here thus are based on several key observations and approaches, as follows.

- We have found that bulky coupling agents and reagents which may work at the primary face are frequently ineffective at the more sterically congested secondary face. Thus, achieving high yields requires avoiding steric conflicts by using linear nucleophiles and less hindered coupling agents, and, when the goal is to attach a bulky group, this is best achieved by first adding a short linker to the secondary face and then attaching the bulky group to the linker. 
- After observing poor results for a number of seemingly straightforward linking reactions, we conjectured that some reactants might act as guest molecules, binding in the CD cavity and preventing reactions from progressing on the rim. This concept motivated the addition of adamantane as an inert guest molecule known to bind $\beta-C D$ with good affinity, with the expectation of displacing the reactant from the cavity; and, indeed, this measure often caused recalcitrant reactions to proceed smoothly.

- Published procedures for isolating $C D$ derivatives are typically complex, requiring chromatography, lyophilization, and/or centrifugation ${ }^{[35-39]}$. We wished to use the simpler approach of purifying the modified CD products by precipitation from DMF with acetone, but our initial attempts failed because, although a visible precipitate formed on addition of acetone to the reaction vessel, the precipitate passed through the finest grades of filter paper. Experiments varying the precipitation conditions revealed that evaporative removal of DMF down to a minimal volume of remaining solution, without allowing precipitation to occur, followed immediately by precipitation with cold acetone, vacuum filtration, and washing with copious amounts of acetone, consistently yielded the pure product. This technique worked for all compounds synthesized in this paper, and no chromatography was required for purification.

The following subsections detail reaction protocols, based on these principles and methods, for facile synthesis of diverse mono-3-substituted CD analogues (Figure 2). The derivatives chosen all represent functional groups that we anticipate will lead to interesting binding properties, through interactions of guest molecules with aromatic, ionic, or hydrogen bonding functionalities. Binding studies of these compounds with various guests will be pursued in future work. 

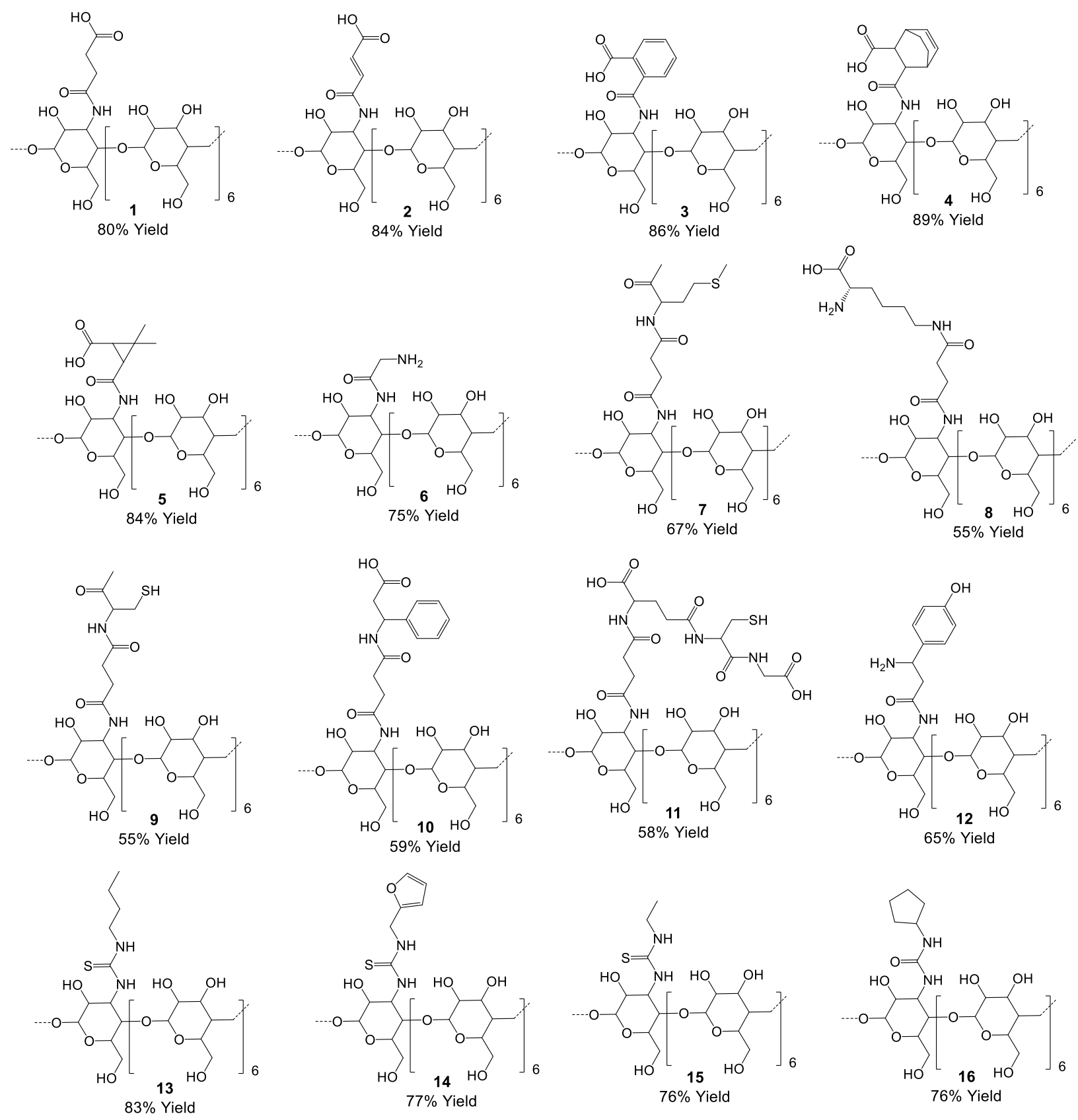

Figure 2. Library of water-soluble, mono-substituted, $\beta$-cyclodextrin analogues synthesised, with final yields as noted.

\section{Amic acids}

Amic acid $\beta-C D$ derivatives are excellent candidates to vary the character of host-guest complexation, because they add both hydrogen bond donor and acceptor groups to the native host. They can be easily synthesized through nucleophilic ring opening of cyclic anhydrides, and the commercial availability of these reagents allows a variety of $C D$ analogues to be synthesized under uniform reaction conditions. The formation of amic acid derivatives 1-5 in yields of $>80 \%$ (Figure 2) was successfully achieved through a one pot strategy that required only stirring at room temperature. This synthesis is based on a primary face 
derivatization of Onagi et al. ${ }^{[40]}$, but we replaced their purification procedure, which requires lyophilization and centrifugation, with the simple acetone precipitation procedure mentioned above. While problems attributed to steric obstruction have been reported for functionalization of the secondary face with bulky groups $^{[25]}$, the high yields produced in this reaction indicate that steric congestion is not a significant obstacle to the formation of the amic acid derivatives, and led us to the concept that linear nucleophiles are preferred in the context of secondary face modification.

\section{Amino Acids}

Biomimetic design is a known approach to developing host molecules for biomedical purposes ${ }^{[25,41]}$. In one method, a host molecule, serving as a scaffold, is decorated with peptide chains ${ }^{[41,42]}$. Indeed, the diverse range of amino acids and their strong binding capabilities make them ideal functionalities for modifying CDs in interesting and potentially biologically relevant ways. Although syntheses of $\beta$-CDs substituted with amino acids have been reported, they have focused on primary face substitution or non-selective secondary face substitution ${ }^{[42,43]}$. Our initial attempts at selective secondary face coupling, with coupling agents HATU, DCC and HBTU, were unsuccessful. However, tetrachloro- $N$-hydroxyphthalimide tetramethyluronium hexa-fluorophosphate (CITU), a recently developed coupling agent for amide synthesis ${ }^{[4]}$, in combination with $\mathrm{N}$-methylmorpholine, allowed synthesis of the glycine adduct, 6, in 75 $\%$ yields. Again, the reaction required only stirring at room temperature, partial drying, and acetone precipitation, followed by a simple Boc-deprotection step and purification by precipitation. Coupling of bulkier amino acids directly to $3-\mathrm{NH}_{2}-\beta-\mathrm{CD}$ remained unsuccessful, though, presumably because of steric hindrance effects, noted above. However, 6 is a good starting point for extension of the peptide chain, as the steric clashes of the polar secondary face are no longer a contributing factor. Accordingly, we have successfully linked Cys and His to the free amino group of $\mathbf{6}$ (data not shown), leading to $\beta-C D$ derivatives with Gly-Cys and Gly-His dipeptide substituents.

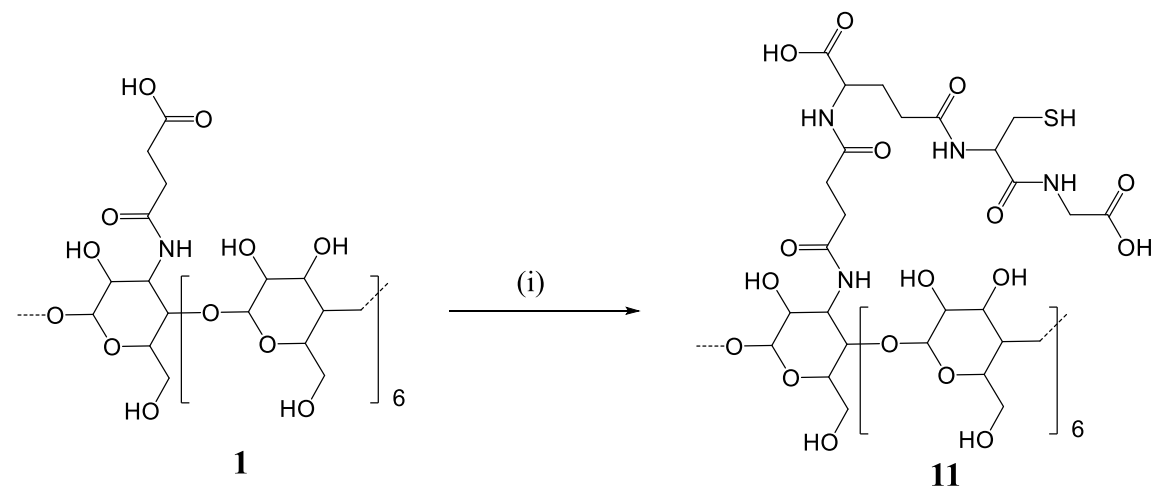

Scheme 1. Synthesis of mono-3-((carboxypropionamido-glutathione)- $\beta$-cyclodextrin (11), (i) CITU, n-methylmorpholine, DMF, $12 \mathrm{~h}$.

Much like the Gly derivative just discussed, the amic acid derivatives described above also represent excellent starting materials for addition of bulky amino acids and peptides to the secondary face. Following the same reaction procedures used for $\mathbf{6}$, we coupled methionine, lysine, cysteine and $\beta$ phenylalanine to 1 , producing $7,8,9$ and 10 at yields $\geq 65 \%$, giving an overall reaction yield of $\geq 55 \%$ from 3- $\mathrm{NH}_{2}-\beta-\mathrm{CD}$. To further validate this approach, we coupled the tripeptide glutathione to $\mathbf{1}$, yielding 11 at $58 \%$ yields (Scheme 1). Again, isolation and purification of $\mathbf{1 1}$ was achieved without the need for 
chromatography. Interestingly, NOESY NMR indicates that the pendant arm does not self-associate inside the CD cavity [Figure S34].

Finally, we conjectured that a third way of coupling bulky amino acids to the secondary rim would be to move away from $\alpha$-amino acids to $\beta$-amino acids, as these should space the bulky side chain further from the secondary rim by the length of an additional methylene group. We tested this for $\beta$-tyrosine, reacting $\beta$-Boc-tyrosine and $3-\mathrm{NH}_{2}-\beta-\mathrm{CD}$ under the same conditions as used to make $\mathbf{6}$. This afforded 12 in $65 \%$ yields, after Boc-deprotection. In contrast, the same reaction with the corresponding $\alpha$-amino acid, tyrosine, proved unsuccessful, presumably because the bulky phenol group sterically hinders reaction with 3- $\mathrm{NH}_{2}-\beta-\mathrm{CD}$. This result further supports the principle that non-hindered linear nucleophiles are necessary for successful addition directly onto the secondary face. The $\beta$-amino acids are appealing substituents because they allow varied side-chains to be positioned particularly close to bound guests.

\section{Ureas and Thioureas}

Ureas and thioureas are frequently employed in the construction of supramolecular host systems, to which they can impart strong hydrogen bonding capabilities ${ }^{[45,46]}$. Syntheses based on urea and thiourea linkages also provide access to further chemical diversity, because isothiocyanates and isocyanates are available with a variety of substituents. Here, the nucleophilic addition of such reactants affords a range of urea and thiourea derivatized $\beta-C D$ analogues, exemplified by 13-16 (Figure 1). These syntheses also offer insight into the determinants of reactivity, as follows.

Initial syntheses of 13 were run at molar ratios of 1:1, 2:1 and 3:1 of isothiocyanate starting material to 3$\mathrm{NH}_{2}-\beta-\mathrm{CD}$, keeping all other reaction conditions constant. The reaction proceeded successfully only when the isothiocyanate reactant was present at 3:1 excess and gave yields $<35 \%$. We hypothesized that the isothiocyanate was acting as a guest molecule, binding inside the $\beta$-CD cavity and hindering progress of the reaction. To explore this hypothesis, we added a competing guest molecule, adamantane, to block the $\beta-C D$ cavity. Adamantane is an inert hydrocarbon, known to have a strong affinity for $\beta-C D^{[12]}$. This approach was successful, as a reaction mixture 1:1:1 of adamantane, $3-\mathrm{NH}_{2}-\beta-\mathrm{CD}$ and isothiocyanate yielded $\mathbf{1 3}$ at $83 \%$ yield. The adamantane was easily removed during the acetone wash purification stage of the workup. Thioureas 14-15 and an analogous urea compound, 16, were also successfully synthesized at high yield (Figure 2 ) by this procedure.

\section{Lack of Aggregation of the Derivatives}

As previously mentioned, the propensity for aggregation of some modified $\mathrm{CDS}^{[47,48]}$ can be problematic. However, for all the compounds reported here, we saw none of the peak-broadening of the ${ }^{1} \mathrm{H}-\mathrm{NMR}$ spectrum that is typically associated with aggregation. The possibility of aggregation can be further examined with diffusion ordered spectroscopy (DOSY) NMR, a technique used to separate NMR signals based on molecular diffusion coefficients. In particular, a molecule that aggregates will yield a lower diffusion coefficient than one of similar molecular weight that remains monomeric. We carried out DOSY studies of $\beta-C D$ analogues from each series created here, amic acid, amino acids, urea, and thiourea, in the presence of native $\beta-C D$ as a control and compared these spectra with that of a covalent $\beta-C D$ dimer (Compound 16, Figure S51), which is larger than any of the derivatives. As shown in Figure 3, the diffusion coefficients of all derivatives studied in this manner fall between those of native $\beta-C D$ and the covalent dimer, which is as expected if the derivatives remain monomeric in solution. 


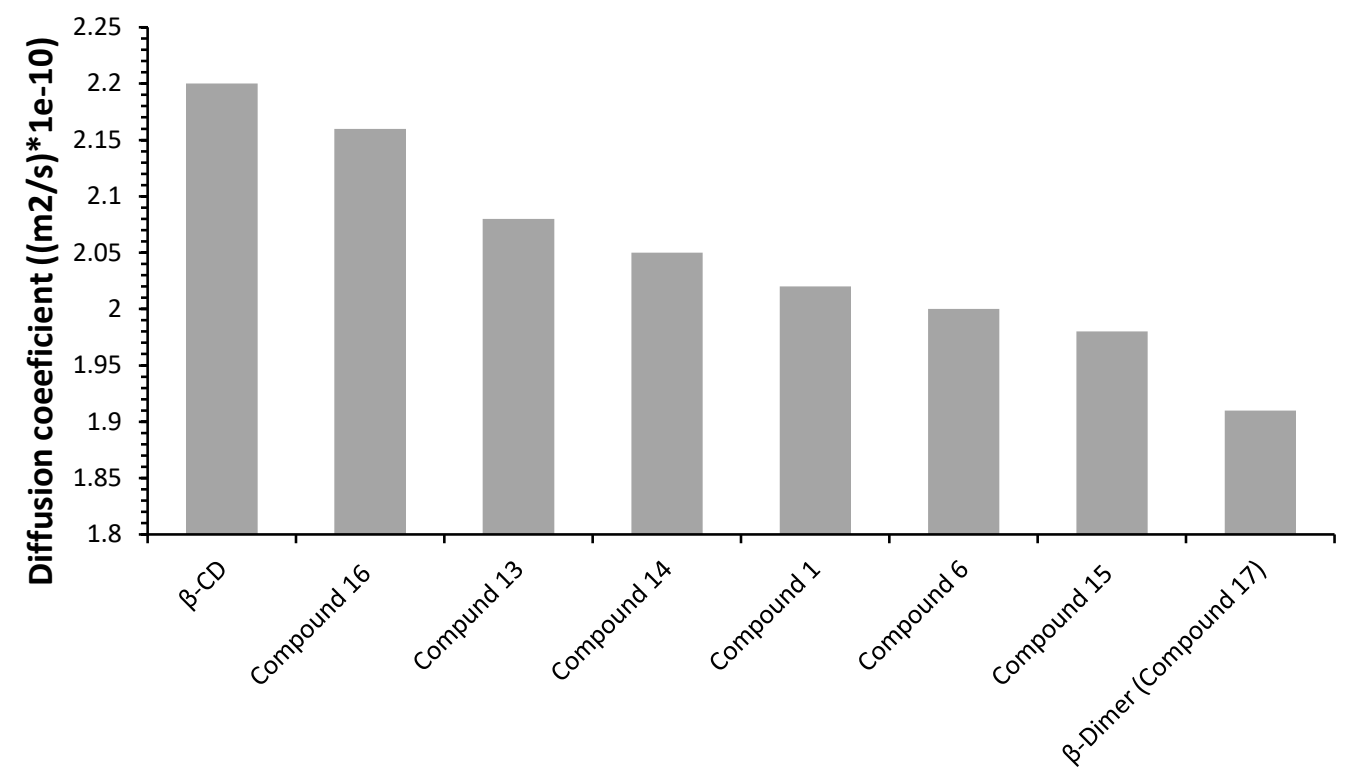

Compound

Figure 3. A. Diffusion coefficients from DOSY NMR, of a selection of synthesised analogues shown in comparison with unmodified $\beta-C D$ and $a-C D$ dimer as controls.

\section{Conclusions}

We have described simple, high-yield, protocols, which require only commonly accessible equipment, to synthesize a wide range of $\beta-C D$ derivatives mono-substituted at the secondary face. These derivatives may be useful in their own right, and they are also scaffolds for further modification, and examples of the far broader array of derivatives that may be accessed by these procedures. Steric hindrance, a key obstacle to functionalizing the secondary face of $\beta-C D$, was circumvented by the use of linear nucleophiles and less bulky coupling agents; by using a skinny linker, such as glycine and amic acids, to position bulkier reactants away from the bulk of the host molecule; and by using $\beta$-amino acids, instead of $\alpha$-amino acids, to position bulky side chains slightly further from the secondary rim. Some reactions were also enhanced by inclusion of adamantane, an inert component of the reaction mixture that presumably helps by displacing reactants from the binding cavity. The synthetic approaches described here should facilitate the optimization of modified $\beta$-CDs for varied applications. They also offer ready access to chemically diverse host-guest complexes to drive the development of accurate force fields for molecular modeling and drug design.

\section{Acknowledgements}

The authors are grateful to Profs. Dionicio Siegel and Thomas Hermann for generously sharing their expertise. NMR spectra were collected at the UCSD Skaggs School of Pharmacy and Pharmaceutical Sciences NMR Facility. We also acknowledge the Molecular Mass Spectrometry Facility at UCSD where the MS-data was obtained. M.K.G. has an equity interest in and is a cofounder and scientific advisor of VeraChem LLC. 


\section{References}

[1] M. E. Davis, M. E. Brewster, Nat. Rev. Drug Discov. 2004, 3, 1023-1035.

[2] T. Loftsson, M. E. Brewster, J. Pharm. Pharmacol. 2010, 62, 1607-1621.

[3] H. M. C. Marques, Flavour Fragr. J. 2010, 25, 313-326.

[4] A. Ciobanu, D. Landy, S. Fourmentin, Food Res. Int. 2013, 53, 110-114.

[5] T. P. Lybrand, J. A. McCammon, G. Wipff, Proc. Natl. Acad. Sci. U. S. A. 1986, 83, 833-835.

[6] W. Chen, C. E. Chang, M. K. Gilson, Biophys. J. 2004, 87, 3035-3049.

[7] N. M. Henriksen, M. K. Gilson, J. Chem. Theory Comput. 2017, 13, 4253-4269.

[8] J. Yin, A. T. Fenley, N. M. Henriksen, M. K. Gilson, J. Phys. Chem. B 2015, 119, 10145-10155.

[9] D. L. Mobley, M. K. Gilson, Predicting Binding Free Energies: Frontiers and Benchmarks, 2017.

[10] L. Wickstrom, P. He, E. Gallicchio, R. M. Levy, J. Chem. Theory Comput. 2013, 9, 3136-3150.

[11] M. V. Rekharsky, M. P. Mayhew, R. N. Goldberg, P. D. Ross, Y. Yamashoji, Y. Inoue, J. Phys. Chem. B 1997, 101, 87-100.

[12] J. Carrazana, A. Jover, F. Meijide, V. H. Soto, J. V. Tato, J. Phys. Chem. B 2005, 109, 9719-9726.

[13] B. Gidwani, A. Vyas, Biomed Res. Int. 2015, 2015, DOI 10.1155/2015/198268.

[14] A. R. Khan, P. Forgo, K. J. Stine, V. T. D’Souza, Chem. Rev. 1998, 98, 1977-1996.

[15] S. Tongiani, D. Vander Velde, T. Ozeki, V. J. Stella, J. Pharm. Sci. 2005, 94, 2380-2392.

[16] E. A. Ottinger, M. L. Kao, N. Carrillo-Carrasco, N. Yanjanin, R. K. Shankar, M. Janssen, M. Brewster, I. Scott, X. Xu, J. Cradock, et al., Curr. Top. Med. Chem. 2014, 14, 330-9.

[17] S. D. Eastburn, B. Y. Tao, Biotechnol. Adv. 1994, 12, 325-339.

[18] D. Rong, V. T. D’Souza, Tetrahedron Lett. 1990, 31, 4275-4278.

[19] R. L. VanEtten, J. F. Sebastian, G. A. Clowes, M. L. Bender, J. Am. Chem. Soc. 1967, 89, 3242-3253.

[20] K. Kellett, S. A. Kantonen, B. M. Duggan, M. K. Gilson, J. Solution Chem. 2018, DOI 10.1007/s10953-018-0769-1.

[21] R. Estrada, G. Vigh, J. Chromatogr. A 2012, 1226, 24-30.

[22] Z. Zawada, A. Tatar, P. Mocilac, M. Buděšínský, T. Kraus, Angew. Chemie Int. Ed. 2018, 57, 1-6.

[23] A. Ueno, R. Breslow, Tetrahedron Lett. 1982, 23, 3451-3454.

[24] D. Hilvert, R. Breslow, Bioorg. Chem. 1984, 12, 206-220.

[25] R. Breslow, S. D. Dong, Chem. Rev. 1998, 98, 1997-2012.

[26] T. Murakami, K. Harata, S. Morimoto, Chem. Lett. 1988, 17, 553-556.

[27] F. Bellia, L. Mendola, C. Pedone, E. Rizzarelli, 2009, 2756-2781. 
[28] S. D. Dong, R. Breslow, Tetrahedron Lett. 1998, 39, 9343-9346.

[29] S. Tian, V. T. D'Souza, Tetrahedron Lett. 1994, 35, 9339-9342.

[30] S. Proniuk, J. Blanchard, J. Pharm. Sci. 2001, 90, 1086-1090.

[31] R. Auzély-Velty, F. Djedaïni-Pilard, S. Désert, B. Perly, T. Zemb, Langmuir 2000, 16, 3727-3734.

[32] J. Stappaerts, T. Do Thi, E. Dominguez-Vega, G. W. Somsen, G. Van den Mooter, P. Augustijns, Int. J. Pharm. 2017, 529, 442-450.

[33] D. Q. Yuan, T. Tahara, W. H. Chen, Y. Okabe, C. Yang, Y. Yagi, Y. Nogami, M. Fukudome, K. Fujita, J. Org. Chem. 2003, 68, 9456-9466.

[34] S. Hanessian, A. Benalil, C. Laferrière, J. Org. Chem. 1995, 60, 4786-4797.

[35] S. Menuel, B. Doumert, S. Saitzek, A. Ponchel, L. Delevoye, E. Monflier, F. Hapiot, J. Org. Chem. 2015, 80, 6259-6266.

[36] K. A. Martin, M. A. Mortellaro, R. W. Sweger, L. E. Fikes, D. T. Winn, S. Clary, M. P. Johnson, A. W. Czarnik, J. Am. Chem. Soc. 1995, 117, 10443-10448.

[37] L. F. Becker, D. H. Schwarz, G. Wenz, Beilstein J. Org. Chem. 2014, 10, 2920-2927.

[38] J. Yoon, S. Hong, 1996, 2792-2795.

[39] N. Vurgun, M. Nitz, Chem. - A Eur. J. 2018, 24, 4459-4467.

[40] H. Onagi, C. J. Blake, C. J. Easton, S. F. Lincoln, Chem. - A Eur. J. 2003, 9, 5978-5988.

[41] M. Boncheva, G. M. Whitesides, Dekker Encycl. Nanosci. Nanotechnol. 2004, 1, 287-295.

[42] P. R. Ashton, R. Königer, J. F. Stoddart, D. Alker, V. D. Harding, J. Org. Chem. 1996, 61, 903-908.

[43] V. Cucinotta, G. Pappalardo, G. Vecchiob, J. Chem. Soc., Chem, Commun. 1991, 293-294.

[44] J. N. deGruyter, L. R. Malins, L. Wimmer, K. J. Clay, J. Lopez-Ogalla, T. Qin, J. Cornella, Z. Liu, G. Che, D. Bao, et al., Org. Lett. 2017, 19, 6196-6199.

[45] A. P. Davis, C. Dias, H. Valkenier, Chem. - A Eur. J. 2018, DOI 10.1002/chem.201800508.

[46] K. Kellett, J. H. Broome, M. Zloh, S. B. Kirton, S. Fergus, U. Gerhard, J. L. Stair, K. J. Wallace, Chem. Commun. 2016, 52, 7474-7477.

[47] A. W. Coleman, I. Nicolis, N. Keller, J. P. Dalbiez, J. Incl. Phenom. Mol. Recognit. Chem. 1992, 13, 139-143.

[48] Y. He, P. Fu, X. Shen, H. Gao, Micron 2008, 39, 495-516. 